\title{
LA SOMATIZACIÓN COMO SÍNTOMA Y COMO SÍNDROME: UNA REVISIÓN DEL TRASTORNO DE SOMATIZACIÓN
}

\author{
JOSÉ LÓPEZ SANTIAGO' Y AMPARO BELLOCH ${ }^{2}$ \\ ${ }^{1}$ Unidad de Salud Mental del Hospital Perpetuo Socorro, Albacete \\ ${ }^{2}$ Departamento de Personalidad Evaluación y Tratamiento Psicológicos, Facultad de Psicología, \\ Universidad de Valencia
}

\section{(Aceptado en marzo de 2002)}

El uso del termino somatización está muy extendido en el campo de la psicopatología y la clínica psicológica y psiquiátrica, a pesar de que su significado y delimitación diagnóstica no están claramente establecidos. El presente trabajo tiene como objetivo profundizar en el estudio del trastorno de somatización. En primer lugar se lleva a cabo una revisión de los distintos significados que se han venido otorgando al término somatización en la literatura científica. Se incluye un análisis de la interpretación de la somatización como un lenguaje de expresión de malestar psíquico, la distinción entre somatización y psicosomática, el estudio del concepto de depresión enmascarada, el concepto de conducta de enfermedad y, por último, se propone la utilización de una definición descriptiva del término somatización. En la segunda parte, el objetivo es examinar el trastorno de somatización como categoría diagnóstica. Se estudian su evolución y validez nosológica, y se presentan propuestas alternativas para la conceptuación del trastorno. Finalmente, se sugieren algunas líneas de investigación que, en opinión de los autores, es necesario desarrollar para lograr una definición más útil y operativa del trastorno de somatización.

Palabras clave: Trastorno de somatización, trastornos somatomorfos, síntomas somáticos funcionales, psicosomática, histeria, síndrome de Briquet.

\section{Somatization as a syndrome and as a symptom: A review of somatization disorder}

The term somatization is largely used in psychopathology as well as in the fields of clinical psychology and psychiatry. However, its meaning and diagnostic characterization are not clearly established. The aim of the present work has been to review the main relevant topics related to the somatization disorder. With this purpose we have analyzed, firstly, the different meanings adscribed to the term, including the following ones: the interpretation of somatization as an expression of psychological distress, the distinction between somatization and psychosomatic disorders, the study of the masked depression concept, and the illness behaviour construct. Secondly, we review the somatization disorder as a diagnostic category, with an emphasis on its evolution and nosological validity. Finally, some suggestions for future research on the somatization disorder are discussed, in order to achieve a more useful conceptualisation of the disorder.

Key words: Somatization disorder, somatoform disorders, functional somatic symptoms, psychosomatics, hysteria, Briquet's syndrome.

La utilización del término «somatización" está bastante extendida, tanto por parte del profesional de la salud mental como en el uso común o habitual del lenguaje. De un modo genérico el término refiere a una queja física, que ocasio- na malestar, en ausencia de hallazgos clínicos que permitan postular una causa orgánica que pueda explicarla completamente.

Desde un punto de vista psicopatologico, la presencia de somatizaciones constituye el denominador común de un grupo de trastornos, incluidos en las actuales clasificaciones nosológicas de 
la CIE-10 (OMS, 1992) y del DSM-IV (APA, 1995) bajo la denominación de trastornos somatomorfos. Dentro de este epígrafe -en los citados manualesencontramos entidades como el trastorno hipocondríaco, el trastorno de conversión, la dismorfofobia y con especial interés para nosotros, el trastorno de somatización, que es, junto al concepto de somatización, objeto de estudio del presente artículo.

Aunque a priori puede resultar claro a qué nos referimos al hablar de somatización o de trastorno de somatización, si analizamos detenidamente los diferentes contextos en los que este vocablo se utiliza, los diversos tipos de quejas a las que se asocia, y las diferentes atribuciones que sobre éstas se realizan, su significado y delimitación se muestran más ambiguos. Son patentes las dificultades conceptuales que pueden encontrarse en el estudio de la somatización y la importancia que tiene el punto de partida que tomemos en su estudio para las posteriores conclusiones que podamos obtener. Así, muy diferente será entender un síntoma determinado como la manifestación o llamada de atención sobre un conflicto subyacente, o entenderlo como fruto de una hipersensibilidad a determinadas lesiones que para otras personas podrían pasar desapercibidas.

El presente artículo intenta adentrarse en el estudio de la somatización tomando como punto de partida la distinción entre somatización como síntoma y como síndrome, es decir, como categoría diagnóstica dentro de las nosologías psiquiátricas. Al hablar de somatización como síntoma el objetivo principal ha sido intentar profundizar en los diferentes usos y significados dados al término por diferentes autores. Respecto a la somatización como categoría diagnóstica, el acento se ha puesto en la evolución nosológica y en la valoración de conceptualizaciones alternativas del trastorno.

\section{EVOLUCIÓN Y SIGNIFICADOS DEL TÉRMINO}

En definitiva, y esto ha ocurrido más de una vez en la historia del pensamiento humano, dar por supuesto que a todo término con un significado ha de corresponder una realidad objetiva precisa, constituye un crasísimo error.

José L. Pinillos (1975, p, 223).

En este epígrafe el principal objetivo será el de intentar explicar y delimitar en qué consiste el trastorno de somatización, más concretamente, a qué se ha querido hacer referencia cuando en la literatura científica se ha usado este término.

Manifestación de un trastorno psíquico en forma de síntoma orgánico

La literatura especializada sobre el tema coincide en señalar a Steckel como el autor que acuñó el término somatización (Chorot y Martínez, 1995; García Campayo, 1999; Kellner, 1991; Martin y Yutzy, 1996), quien le otorgó un significado similar al del concepto de conversión de Freud, es decir, el de un mecanismo que convierte la «energía psíquica» en síntomas "psicógenos" -en el caso de las somatización, físicos- (Freud, 1993; Laplanche y Pontalis, 1994). Steckel (1943) definió el término cómo: «el proceso en que un trastorno psicológico puede producir trastornos corporales» (citado en García Campayo, 1999). Probablemente sea ésta definición, que posee una fuerte connotación etiológica y una estrecha vinculación, más o menos explícita, con los marcos teóricos psicodinámicos, la que 
mayor influencia ha tenido en la interpretación del término $\mathrm{y}$, por extensión, de aquellos síntomas físicos a los que el médico no ha podido relacionar con una causa orgánica que pueda justificarlos (Kellner, 1985, 1986; Lipowski, 1988; Martin y Gutzy, 1996).

Este modo de entender la somatización ha sido mantenido, con algunas matizaciones, por diversos autores (p.ej., Bridge y Golberg, 1985; Ford, 1983; Katon, Kleinman y Rosen, 1982; Kleinman y Kleinman, 1986, Lipowski, 1986). Una definición que con frecuencia se cita en la literatura reciente para definir el trastorno es la propuesta en Kleinman y Kleinman (1986), para quienes la somatización es "la expresión de malestar social y personal mediante un idioma de quejas somáticas que lleva a la búsqueda de ayuda médica».

Resumiendo de una forma operativa este concepto de somatización, Bridge y Goldberg (1985) han definido al paciente somatizador - diferenciándolo del que denominan «psicologizador»-a partir de la presencia de cuatro características. Según ellos, un somatizador sería un individuo que: 1) realiza una presentación somática de su malestar; 2) realiza una atribución somática para sus síntomas, 3) se constata la existencia de un trastorno psiquiátrico; y 4) existe la expectativa de una respuesta positiva al tratamiento psiquiátrico.

El peso y la utilización de esta conceptualización pueden quedar de manifiesto al observar cómo en el uso en castellano del término se recoge este significado. Los dos "diccionarios de uso" más importantes de la lengua castellana (María Moliner, 1998 y Manuel Seco, 1999; el diccionario de la RAE no incluye esta voz) coinciden en su acepción, definiendo la somatización como el proceso de "convertir un trastorno psíquico en alteraciones orgánicas o funcionales. Manifestarse un trastorno psíquico en forma de síntomas orgánicos o funcionales» (Moliner, 1998).

Esta conceptuación ha recibido diversas críticas, a pesar de ser, como hemos indicado, de frecuente uso y estar bastante aceptada por parte de la comunidad científica. Las críticas se han centrado principalmente en cuestionar este tipo de explicaciones por basarse en supuestos teóricos discutibles (Kellner, 1986, 1991; Martin y Yutzy, 1996) y por poner el acento en hipotesis etiológicas con escasa fundamentación empírica (Kirmayer y Taillefer, 1996; Lipowski, 1988).

Desde esta definición de somatización como un lenguaje de expresión de malestar psicológico, el etiquetar una queja como tal puede invitar a pensar que ésta es imaginaria, o que de un modo más o menos consciente es provocado por la persona que la refiere. Sin embargo, según diversos autores (García Campayo, 1999; Kellner, 1986; Kirmayer y Taillefer, 1996) es probable que muchos síntomas y síndromes sean debidos a pequeñas lesiones o sutiles alteraciones, difíciles de detectar por pruebas convencionales de laboratorio. $Y$ aunque la somatización se distingue claramente de la simulación, es muy frecuente que en los medios sanitarios, e incluso en la opinión general, se tienda a estigmatizar a aquella persona que presenta frecuentes y diversas quejas físicas sin una clara justificación médica. Es decir, como Kirmayer y Taillefer (1996) plantean, para la medicina occidental «aquello que es físico, es más real, sustancial y en último término más legítimo entenderlo como enfermedad que como psicológico» (p. 333) y por tanto desde la concepción dualista "mente-cuerpo", predominante en la medicina occidental, resulta difícil entender como "normal» que existan síntomas somáticos sin un referente orgánico claro que los "explique». En consonancia con esta idea llama la aten- 
ción cómo para Chodoff, (1974; citado en Lipowski, 1988) una de los posibles significados del término somatización es el de una palabra coloquial utilizada para designar algo indeseable, es decir, presentar quejas somáticas que no puedan ser justificadas orgánicamente puede convertirse en algo indeseable.

\section{La somatización y lo psicosomático}

Somatización y psicosomático son conceptos íntimamente relacionados, ambos hacen referencia a manifestaciones generalmente bien diferenciables, pero que en ocasiones son confundidas o tomadas como sinónimos. Los dos términos expresan una relación entre lo físico y lo psíquico pero desde diferentes puntos de vista. Siguiendo a Kellner (1991), «el término psicosomático indica una relación mente-cuerpo. Esta consta de un proceso psicofisiológico así como de una enfermedad física que puede estar influido por factores psicológicos (p.ej. asma bronquial o úlcera péptica). Los síndromes o trastornos psicosomáticos son enfermedades físicas en las cuales los procesos emocionales pueden jugar un papel en algunos pacientes». Por tanto, la diferencia fundamental estriba en que en los trastornos psicosomáticos sí se constata una dolencia física, junto con la posibilidad de que factores psicológicos puedan vincularse con su aparición, mantenimiento o agravamiento. Por su parte, en la somatización no aparece un claro diagnóstico médico (i.e., orgánico) que pueda justificar esos síntomas.

Según Kellner (1991) podemos afirmar que en diversas ocasiones la utilización de los términos psicosomático y somatización ha sido confusa y se han usado arbitrariamente para referirse a aquellos síntomas, síndromes o pacientes en los que el investigador o especia- lista (sea médico, psicólogo o cualquier otro profesional) ha percibido como interrelacionados aspectos psicológicos y somáticos. Como Valdés (1991) pone de manifiesto, "en la practica médica cotidiana, se considera trastorno psicosomático a todo síntoma o molestia que no encaja en ninguna entidad nosológica y que, a falta de hallazgos biológicos justificativos, prejuzga la participación etiopatogénica de "factores psíquicos». Paradojicamente, este autor incluye los trastornos somatomorfos en un capítulo sobre Trastornos psicosomáticos (véase Introducción a la psicopatología y psiquiatría, Vallejo, 1991).

\section{La somatización cómo un síntoma} de depresión

Muy frecuentemente la somatización ha sido asociada con el trastorno depresivo (Escobar Burnam, Karno, Forsythe y Golding, 1987; Hällström, 1998; Katon, Lin, Von Korff, Lipscomb y Bush, 1991; Kellner 1986; OMS, 1992; Portegijs, 1996; Simon y VonKorff, 1991), hallándose una importante relación tanto para población psiquiátrica (p.ej. Hiller, Rief y Fichter, 1997) como en atención primaria (p.ej. Kirmayer y Robbins, 1996). Es destacable el hecho de que pacientes somatizadores tienden a padecer más trastornos psiquiátricos que pacientes con síntomas físicos de enfermedad (García Campayo et al., 1995; Kisely, Goldberg, Simon, 1997); a la vez que los pacientes depresivos presentan un mayor índice de síntomas somáticos que pacientes no depresivos (Escobar, Waitzkin, Silver, Gara y Holman, 1998; Gureje, Ustum y Simon, 1997). Es decir se constata una correlación positiva entre depresión y síntomas somáticos (Kellner, 1986, 1991; Martin y Yutze, 1996).

De esta relación y sus corolarios téricos surge el término de depresión 
enmascarada. Por este término se entiende la manifestación de un trastorno depresivo fundamentalmente mediante síntomas físicos, siendo éstos más intensos y graves que los síntomas psíquicos (Bridge y Goldberg, 1985; Hällström, 1998), quedando los síntomas emocionales y cognitivos atenuados, ocultados o negados (Kirmayer y Taillefer, 1996). Ello da lugar, para los autores que defienden esta conceptualización de la somatización, a la presencia de trastornos depresivos que generalmente no son diagnosticados, y por consiguiente quedan sin tratamiento en un marco de atención a la salud mental.

En el estudio de Hällström (1998) se entiende por depresión enmascarada un trastorno depresivo (depresión mayor o distimia) que es únicamente diagnosticado tras una detallada evaluación psiquiátrica de pacientes que puntuan alto en presencia de síntomas somaticos $y$ alexitimia, siendo ignorado el diagnóstico en una primera evaluación rutinaria por médicos de atención primaria. Por tanto, el autor habla de depresión enmascarada cuando se constata la existencia de un cuadro depresivo que había sido pasado por alto por los médicos de atención primaria, en paciente con frecuentes síntomas somáticos no explicados.

Este hecho nos lleva a pensar que el término de depresión enmascarada puede ser probablemente más una cuestión de evaluación y diagnóstico (o ausencia de éste), que un problema referido a la existencia de una categoría diagnóstica o tipología psicopatológica diferente. Es importante tener en cuenta que, en la mayoría de las ocasiones, los pacientes acuden como primera consulta de su malestar, ya sea éste físico o de otro tipo, a la consulta del médico de atención primaria, siendo éste un marco que favorece indiscuti- blemente que las quejas del paciente se centren en los aspectos somáticos, aunque el malestar pueda ser de otro tipo ( mental, psicosocial, etc.)

Según un trabajo de Kirmayer y Robbins (1996), únicamente un $21 \%$ de los pacientes que presentan un trastorno ansioso o depresivo comunican síntomas mentales a su médico de cabecera. Es decir, que preferentemente refieren síntomas físicos, lo que no significa que no exista otra sintomatología de carácter cognitivo. Según las conclusiones de este interesante trabajo, sólo una pequeña proporción de los pacientes inicialmente diagnosticados como somatizadores (en el sentido de manifestar un trastorno psíquico enmascarado tras síntomas físicos), "se resisten a una interpretación psicológica de su malestar», después de someterse a una adecuada evaluación diagnóstica. Es decir, pocos son los que siguen considerando que sus síntomas están causados indudablemente por una enfermedad física. Concluyen que, en el contexto de atención primaria, presentar fundamentalmente síntomas orgánicos viene determinado «más por el contexto social de la atención médica que por las características psicológicas del pacienten (p. 948).

La comorbilidad entre somatización y otros trastornos psiquiátricos, en concreto depresión y trastornos de ansiedad, como hemos indicado, ha sido en la mayoría de los estudios muy elevada, constatándose en población española (García Campayo, Campos, Pérez-Echeverría y Lobo, 1996a) una comorbilidad por encima del $85 \%$ (criterios DSM-IIIR). Estos datos invitan a pensar que lo que en ocasiones se denomina somatizador puede tratarse de un trastorno ansioso o depresivo que presenta manifestaciones físicas, síntomas que constituyen una característica intrínseca del propio trastorno tímico o de ansiedad (APA 1995, OMS, 1992), y que general- 
mente se presentan acompañados de sus correlatos cognitivos y emocionales (Kellner, 1991, Kirmayer y Robbins, 1996). Resulta prioritaria la identificación y diagnóstico de esos trastornos, además de un adecuado diagnóstico diferencial (Lidbeck, 1997; Vieta, Gasol, Blanch y Civera, 1996), más que a atribuir a la somatización un mecanismo de «enmascaramiento» de otras patologías. (Kirmayer y Robbins, 1996).

\section{La somatización como conducta de enfermedad}

Cuando se utiliza el término somatización, generalmente no sólo se quiere hacer referencia a la presencia de síntomas somáticos, sino que el acento muy probablemente se está poniendo en las "conductas de enfermedad" que supuestamente el paciente somatizador está presentando (ver p.ej. Kleinman y Kleinman, 1986). El término "conducta de enfermedad» (illness ${ }^{1}$ behavior) fue acuñado por David Mechanic en la década de los 60 para hacer referencia a una conducta relevante respecto a cualquier condición que cause, o pueda causar habitualmente, que una persona se preocupe por sus síntomas y busque ayuda (Rodríguez Marín, 1995). Pilowsky (1969) acuñó el término más específico de "conducta anormal de enfermedad", explicitando su significación dentro del contexto de los trastornos somatomorfos (Chorot y Martínez, 1995). La conducta de enfermedad se considera anormal cuando existe una discrepancia entre la patología orgánica observada y la respuesta que manifiesta el paciente.

El concepto de "conducta de enfermedad" implica una serie de manifesta-

\footnotetext{
${ }^{1}$ El término illness hace referencia al malestar subjetivo frente al vocablo disease con el que se designa la lesión objetiva ocasionada por la enfermedad.
}

ciones que están en consonancia con las características atribuidas al rol de enfermo, tales como las verbalizaciones de malestar, frecuentes visitas al médico, consumo de medicamentos, permanecer en casa sin acudir al trabajo, etc. (Chorot y Martínez, 1995), características que usualmente se han asociado con el concepto de somatización y han estado unidas indisolublemente con él (Bridge y Golberg, 1985; Lipowski, 1988; Kellner, 1985; OMS; 1992). Además, se acuña el término "doctor shoping" para designar el comportamiento reiterado de los grandes consumidores de recursos sanitarios, sin que exista justificación (orgánica) para ello, lo que desemboca en no pocas ocasiones en la injuriosa denominación de "malos pacientes» o, en el mejor de los casos, "pacientes difíciles». Consideramos que equiparar el concepto de somatización al de conducta de enfermedad puede resultar estigmatizante para las personas que son etiquetadas con este diagnóstico, y, lo que es peor, puede repercutir negativamente en su evolución y tratamiento. Este aspecto ha sido asimismo denunciado por diversos autores ya mencionados (p.ej., Z. J. Lipowski, L. Kirmayer o R. Kellner).

\section{Somatización como término}

descriptivo: Síntomas somáticos no explicados médicamente

Como hemos analizado a lo largo de los epígrafes anteriores, bajo el vocablo somatización encontramos siempre significados, etiologías o conceptuaciones que, de forma más o menos enmascara$d a$, sobrepasan el hecho descriptivo de la manifestación de síntomas somáticos. Esto ha llevado a diversos autores a preferir la denominación puramente descriptiva de "síntomas no explicados médicamente», frente a los términos 
somatización o síntomas somáticos funcionales (véanse por ejemplo, Deary, Scott y Wilson, 1996; Kirmayer y Taillfer, 1996; Kroenke, Spitzer, de Gruy, Hahn, Linzer, Williams, Brody y Davies, 1997). Esta denominación simplemente implica la existencia de tales síntomas, omitiendo cualquier referencia de carácter etiológico o teórico, al menos de forma generalizada.

Lipowski, cuyo trabajo ha tenido una destacada influencia en la conceptuación del trastorno, criticaba en su muy citado artículo Somatization: The concept and its clinical application (1988) aquellas definiciones que, a partir de Steckel, habían dado una visión de la somatización centrada en supuestos mecanismos de defensa y procesos inconscientes, que proporcionaban una clara funcionalidad a los síntomas. Otros autores, (p.ej., Kellner, 1986, 1991), han continuado utilizando los términos de somatización o síntomas somáticos funcionales, pero explicitando que su uso tiene un carácter descriptivo y no implica que los síntomas sean utilizados para ningún fin por parte del paciente, es decir, que no atribuye ninguna funcionalidad a los mismos. Kellner abogaba por una definición operativa basada en el significado que la APA (concretamente, en el DSMIII-R) otorgaba al término de trastorno somatoforme indiferenciado (Kellner, 1991, 1994), es decir: "uno o más síntomas físicos [...] en los que una exploración apropiada no descubre ningún tipo de patología orgánica o de mecanismo fisiopatológico que explique las molestias [...] o, cuando existe una patología orgánica, los síntomas o el deterioro social o profesional resultante son claramente deşproporcionados" (APA, 1988, pp. 319-320)

Resumiendo la posición de Kellner (1985, 1986, 1991), uno de los autores más destacados en el estudio de la somatización, se puede concluir que la exis- tencia de síntomas funcionales no puede ser explicada por una única teoría ni un único proceso patológico, siendo ésta un fenómeno extremadamente complejo y determinado multi-factorialmente, y que además está presente en diversas categorías diagnósticas.

\section{NOSOLOGÍA DEL TRASTORNO DE SOMATIZACIÓN}

La constatación clínica de pacientes con múltiples y recurrentes quejas físicas para las que no es posible hallar un origen orgánico, y por tanto cabe interpretar como resultado de una "enfermedad mental», tiene una larga historia. En 1859 Paul Briquet, en su obra Traite Clinique et Therapeutique de L'histerie, describió un cuadro psicopatológico principalmente presente en mujeres y caracterizado por frecuentes quejas sexuales y síntomas de dolor. El síndrome era conceptuado como una forma de histeria en la que los pacientes presentaban una conducta extravagante y emocional (Chorot y Martínez, 1995). El trastorno aparecía a una edad temprana y se mantenía estable a lo largo del tiempo (Simon y Gureje, 1999). La descripción de Paul Briquet de este síndrome clínico ha sido la base a partir de la cual se ha ido configurando, a través de los diferentes sistemas diagnósticos, el actual Trastorno de Somatización. El concepto fue retomado en el DSM II de 1968, donde bajo la denominación de Síndrome de Briquet, se describe un subgrupo de pacientes histéricos caracterizados por múltiples quejas somáticas principalmente referidas a vómitos, intolerancia a la comida, pérdida de conciencia, cambio de peso, dismenorrea e indiferencia sexual (Chorot y Martínez, 1995).

El principal impulso para clarificar y delimitar unos criterios que facilitaran el diagnóstico del trastorno se da en los 
años sesenta, gracias al trabajo de Samuel B. Guze y sus colaboradores del denominado grupo de San Luis (Chorot y Martínez, 1995). Bajo la denominación de histeria o Síndrome de Briquet definen un trastorno caracterizado por la existencia de un patrón recurrente de síntomas pertenecientes a diferentes sistemas orgánicos y asociados con malestar psicológico, no pudiendo asociarse a una enfermedad física que los justifique adecuadamente (Cloninger, Martin, Guze, Clayton, 1986; Guze y Perley, 1963; Perley y Guze, 1962). Para estos autores el diagnóstico del síndrome de Briquet requería la presencia de al menos 25 síntomas de un listado de 60, pertenecientes a 9 o 10 categorías. Estos criterios posteriormente fueron incluidos, bajo la denominación de "histeria», dentro de los criterios diagnósticos para trastornos psiquiátricos de Feighner et al. (1972).

Es en el DSM-III (APA, 1980) cuando por primera vez se utiliza el término trastorno de somatización como diagnóstico oficial, cuya conceptuación es directa- mente heredera de los criterios del grupo de Feighner (Liu, Clark y Eaton, 1997; Martin y Yutzy, 1996; Tomasson, Kent y Coryell, 1993). La sustitución del término síndrome de Briquet por el de trastorno de somatización viene motivada por el interés de suprimir, en esa versión del manual diagnóstico, la mayor parte de epónimos (Martin y Yutze, 1996). Es también en el DSM-III donde por primera vez se incluye la categoría de trastornos somatomorfos, que en los aspectos esenciales mantiene su contenido hasta el actual DSM-IV.

El trastorno por somatización queda definido, con un carácter esencialmente descriptivo, como un desorden crónico (aunque fluctuante), polisintomático, que empieza antes de los de los 30 años y que ocurre prioritariamente en la mujer. El número de síntomas evaluados pasa de los 60 del listado original a 37, excluyéndose, entre otros, los síntomas de carácter psicológico (Tomasson et al., 1993), como por ejemplo: nerviosismo, sentimientos depresivos, facilidad de llanto o pensamientos suicidas (veáse la Tabla 1).

Tabla 1. Listado de síntomas de Feighner para la histeria (síndrome de Briquet)

Grupo 1: Cefaleas, enfermizo la mayoría de la vida

Grupo 2: Parálisis, afonía, ataques o convulsiones, inconsciencia, amnesia, sordera, alucinaciones, retención urinaria, dificultades de la marcha, otros síntomas "neurológicos"

Grupo 3: Fatiga, sensación de nudo en la garganta, voz débil, visión borrosa, debilidad, disuria.

Grupo 4: Respiración dificultosa, palpitaciones, crisis de angustia, dolor torácico, mareos.

Grupo 5: Anorexia, pérdida de peso, fluctuaciones importantes de peso, nauseas, distensión abdominal, intolerancias alimentarias, diarrea, estreñimiento.

Grupo 6: Dismenorrea, irregularidades menstruales, amenorrea, menstruaciones abundantes.

Grupo 7: Indiferencia sexual, frigidez, dispareunea, otras dificultades sexuales, vómitos durante los 9 meses de embarazo o hiperemesis grávida.

Grupo 8: Dolor abdominal, vómitos.

Grupo 9: Dolor de espalda, dolor articular, dolor de las extremidades, dolor o escozor en los órganos sexuales, boca o recto; otros dolores corporales.

Grupo 10: Nerviosismo, miedos, sentimientos depresivos, necesidad de abandonar el trabajo o incapacidad para realizar las tareas habituales de la casa a causa de sentirse enfermo, facilidad de llanto, sentimiento de desespero, pensamientos positivos frente a la muerte, necesidad de morirse, pensamiento suicida e idea suicida 
Este grupo de 37 síntomas lo componen aquellos que mejor discriminan entre el síndrome de Briquet y otros trastornos psiquiátricos como ansiedad y depresión (Yutzy et al., 1995) y «deben afectar invariablemente» a los siguientes sistemas orgánicos: pseudoneurológico, gastrointestinal, sistema reproductor femenino, psicosexual, dolor y cardiopulmonar. La corroboración de que el síntoma está «realmente presente» no es imprescindible: «el hecho de que el síntoma sea narrado por el individuo ya es suficiente» (APA, 1980, p. 255). Para recibir el diagnóstico se requiere la presencia de una historia de al menos 12 síntomas en hombres y 14 para las mujeres, es decir, se establece un criterio diferente en función del sexo. (Es importante tener en cuenta que entre los síntomas se incluyen algunos propiamente femeninos, como son por ejemplo tener dolor durante la menstruación o vómitos en el embarazo.)

Con el DSM-III-R (APA, 1988), vuelve a igualarse el número de síntomas para hombres y mujeres, siendo ahora 13 los necesarios para poder realizar el diagnóstico, y el número de síntomas a evaluar se reduce de 37 a 35 (Yutzy et al., 1995). Otra importante modificación es la inclusión de la creencia de estar enfermo como criterio primario y una alternativa a la presencia de múltiples síntomas. En concreto el Criterio $A$ indica: «Historia de múltiples síntomas o creencia de estar enfermo" (p. 315). Característica diagnóstica ésta que no será mantenida en el DSM-IV. También se explicita que los síntomas no aparecen únicamente durante las crisis de angustia y se incluye también como síntoma diagnóstico aquel que, aún poseyendo un origen médico, ocasiona un malestar excesivo al que cabría esperar por la historia clínica. Finalmente el DSM-III-R propone 7 síntomas que podrían considerarse casi patognomóni- cos. Estos síntomas coinciden, y probablemente proceden, de los propuestos como criterios de screening para el Trastorno por Somatización por Ohmer y Desouza (1985). Según estos autores la presencia de dos o más síntomas de este grupo de 7 (dismenorrea, nudo en la garganta, vómito, dificultades para respirar, ardor en recto, genitales o boca, dolor en extremidades y amnesia), proporciona un diagnóstico preciso del trastorno. Este screening ha sido validado en versión española por García Campayo, Sanz, Pérez-Echeverría, Campos y Lobo (1996b). En este trabajo el punto de corte es de tres o más síntomas. Según los autores, las diferencias se explican por la utilización de criterios metodológicos distintos y por las diferencias culturales entre ambas muestras.

En los criterios para el trastorno de somatización propuestos en el DSM-IV (APA, 1994) se ha pretendido realizar una definición más útil y operativa, tanto desde el punto de vista de la especificidad y sensibilidad, como de la facilidad y comodidad a la hora de establecer el diagnóstico (Martin y Yutzy, 1996; Yutzy et al., 1995). El cambio más destacado respecto a las anteriores versiones del DSM hace referencia al número de síntomas, quedando ahora el criterio reducido a 8 y manteniéndose el punto de vista del DSM-III-R al equipararlo en ambos sexos. Además, los síntomas han de especificarse, requiriéndose al menos una historia de 4 síntomas de dolor, 2 gastrointestinales, 1 síntoma sexual y 1 síntoma pseudoneurológico. También se ha eliminado el criterio referido a la creencia de tener una enfermedad, junto con la referencia explícita al carácter de screening de algunos síntomas.

Otro aspecto destacado en la definición del DSM-IV es el requerimiento de que los síntomas provoquen deterioro significativo en áreas importantes de la actividad de la persona o que estos con- 
duzcan a la búsqueda de atención médica (criterio C).

Respecto a la CIE, el concepto de trastorno de somatización no fue incluido hasta su décima versión (OMS, 1992), siendo conceptuado por la CIE-9 como un tipo de histeria, sin especificar criterios para el diagnóstico (Tomasson et al, 1993). Para este sistema clasificatorio el criterio fundamental es la presencia de síntomas somáticos múltiples y variables para los que no se ha encontrado explicación médica (no especifica como han de distribuirse estos aunque destaca como los más frecuentes molestias gastrointestinales y dérmicas junto con quejas menstruales y sexuales). Incluye también como criterio, que constituye denominador común de los trastornos somatomorfos, «la negativa persistente a aceptar las explicaciones o garantías reiteradas de médicos diferentes de que no existe una explicación somática para sus síntomas" (OMS, 1992, p. 203). Y por último estos síntomas o "el comportamiento consecuente" ocasionan cierto grado de deterioro en el comportamiento social y laboral.
Dos son las principales diferencias que podemos encontrar en las definiciones que las dos clasificaciones vigentes de los trastornos mentales (CIE-10 y DSM-IV) ofrecen del trastorno de somatización. En primer lugar la CIE-10 no explícita ni la necesidad de un número concreto de síntomas ni una distribución determinada de estos, cosa que el DSM-IV, en su mayor interés de ofrecer definiciones operativas de los desórdenes que incluye, sí especifica. En segundo lugar, y probablemente con mayor significación psicopatológica, encontramos la diferencia que ambos sistemas presentan con relación al criterio referido al rechazo del paciente del diagnóstico médico, característica no mencionada en el DSM-IV.

En los criterios diagnósticos de investigación de la CIE-10 (OMS, 1993) sí se propone una distribución específica de síntomas, requiriendo la presencia de 6 síntomas de un total de 14 (ver tabla 2), distribuidos en cuatro categorías. Estos 6 síntomas deben pertenecer al menos a dos de las categorías. El criterio de rechazo persistente a aceptar el diagnós-

Tabla 2. Síntomas de los criterios de investigación de la CIE-10 para el trastorno de somatización

\section{Síntomas gastrointestinales:}

1. Dolor abdominal

2. Náuseas

3. Mal sabor de boca o lengua saborral

4. Sensación de plenitud abdominal o meteorismo

5. Queja de vómito o regurgitación

6. Quejas de tránsito intestinal rápido o diarreas mucosas

\section{Síntomas cardiovasculares:}

7. Falta de aliento sin hacer esfuerzos

8. Dolor torácico

\section{Síntomas urogenitales:}

9. Disuria o quejas de micción frecuentes

10. Sensación desagradable en o alrededor de los genitales

11. Queja de flujo vaginal excesivo o desacostumbrado

Śntomas cutáneos o de dolor:

12. Erupciones o decoloración de la piel

13. Dolor en los miembros, extremidades o articulaciones

14. Sensaciones desagradables, falta de tacto o parestesias

Nota: Fuente OMS (1993). 
tico médico de ausencia de enfermedad, permanece en esta clasificación. Frente a los listados de síntomas propuestos por las diversas conceptuaciones del DSM, la ofrecida en los criterios de investigación de la CIE-10 presenta un aspecto de interés que es la reducción considerable del número de síntomas a evaluar.

Desde las primeras descripciones clínicas del síndrome, han sido muchas las modificaciones que se han hecho a lo largo de los años, lo que puede llevar a dudar de sí bajo los diferentes diagnósticos se esconde el mismo trastorno. En un trabajo de Yutzy et al. (1995) diseñado para evaluar la validez de los criterios propuestos por el DSM-IV, se ha encontrado una alta concordancia entre los diferentes criterios del trastorno de somatización (DSM-III, DSM-IIIR y DSM-IV) y a su vez entre éstos y los criterios de Perley y Guze (1962). Contrariamente a estos resultados, la equivalencia hallada entre el DSM y los criterios desarrollados en la CIE-10 es muy escasa.

Estos resultados otorgan cierta solidez al trastorno de somatización, entendido de forma genérica como un trastorno polisintomático y persistente durante varios años. Además, permiten enlazar, en un sentido amplio, el síndrome de Briquet (criterios de Perley y Guze, 1962) con el trastorno de somatización, ya que a pesar de las sustanciales diferencias, hay diversos estudios que proporcionan considerables correlaciones entre ambos: $0,59,0,60$ y 0,84 , (Brown y Smith, 1991; Tomasson, et al., 1993; Yutzy et al, 1995, respectivamente). Sin embargo, esta concordancia diagnóstica ya no resulta tan significativa si incluimos dentro de la definición del trastorno aspectos etiológicos o atribucionales respecto a la naturaleza y origen de los síntomas (Kirmayer y Robbins, 1996). En la Tabla 3 se resumen las principales diferencias en torno al diagnóstico del Trastorno de Somatización en las diversas versiones recientes de los sistemas diagnósticos de la APA y la OMS, así como en el planteamiento de Perley y Guze.

\section{Aspectos de debate sobre los criterios diagnósticos}

Algunas de las características diagnósticas que actualmente se incluyen en el trastorno de Somatización, son objeto de debate. Esto queda patente en las diferencias, señaladas en el epígrafe anterior, entre los dos sistemas diagnósticos vigentes en la actualidad y en las modificaciones que se han producido en sus sucesivas versiones.

Por lo que se refiere a la característica definitoria del trastorno, es decir, una historia múltiple de síntomas somáticos, tres han sido los ejes de discusión en cuanto a la conceptuación de los síntomas. En primer lugar, ha habido una tendencia a disminuir el número de síntomas considerados como somatizaciones, eliminándose principalmente los de carácter psicológico. A pesar de este aspecto común, los listados de síntomas proporcionados por el DSM-IV y los de CIE-10 (criterios de investigación), resul$\tan$ muy divergentes. Un segundo aspecto se refiere a la progresiva disminución del número de síntomas necesarios para realizar el diagnóstico, pasándose de los 25 de la definición del Síndrome de Briquet a los 8 propuestos por el DSM-IV. Finalmente, aunque en las diferentes versiones del DSM los sintomas han estado organizados en categorías, es en el DSMIV donde se solicita como criterio diagnóstico una distribución específica de los síntomas en al menos cuatro apartados diferentes (descritos previamente). Esta distribución de síntomas en subgrupos ha sido cuestionada recientemente (Liu et al., 1997). 


\begin{tabular}{|c|c|c|c|c|c|c|c|c|c|}
\hline \multirow{2}{*}{ 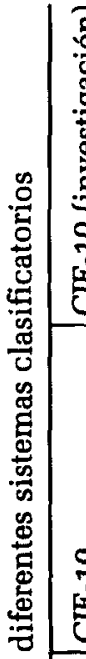 } & 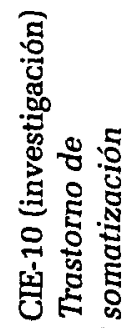 & 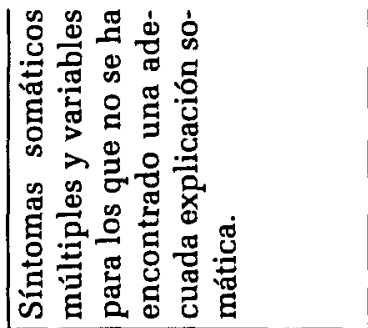 & 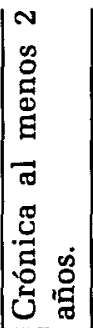 & 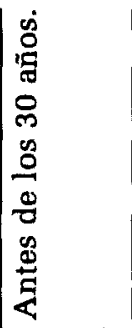 & 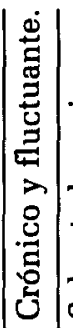 & 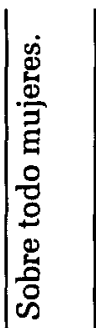 & 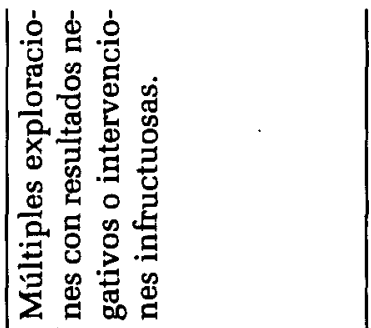 & 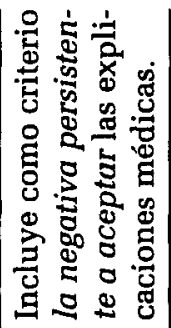 & $\begin{array}{l}\vec{J} \\
\stackrel{\Delta}{ } \\
0\end{array}$ \\
\hline & 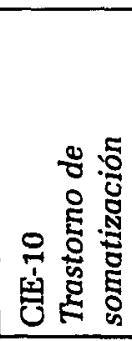 & 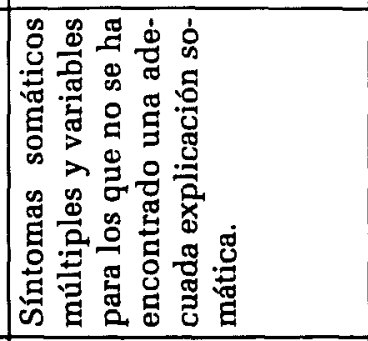 & 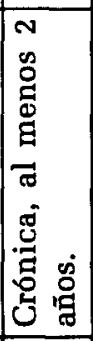 & 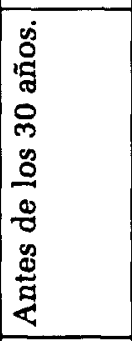 & 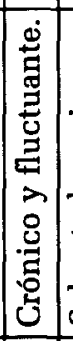 & 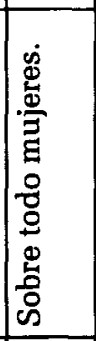 & 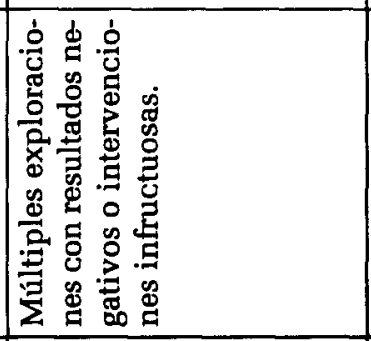 & 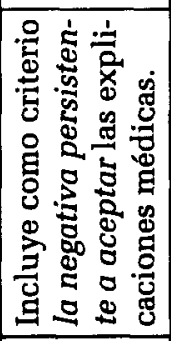 & 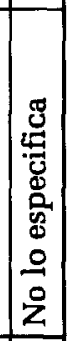 \\
\hline & 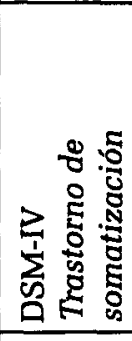 & 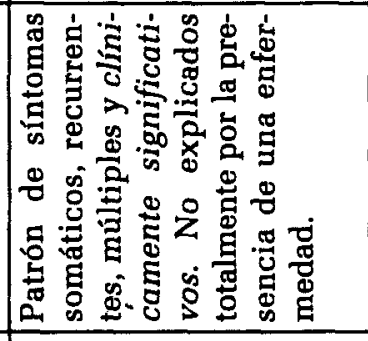 & 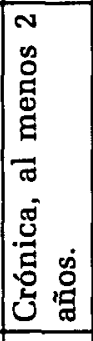 & 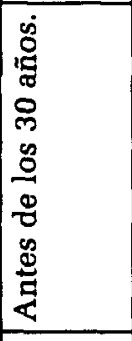 & 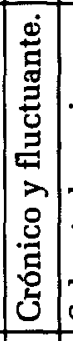 & 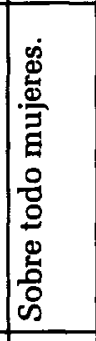 & 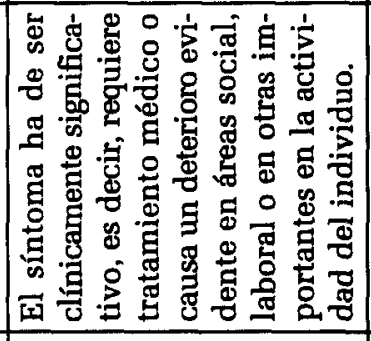 & 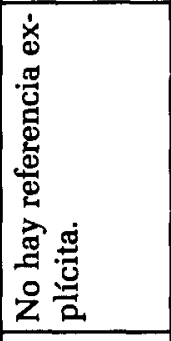 & 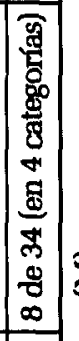 \\
\hline & 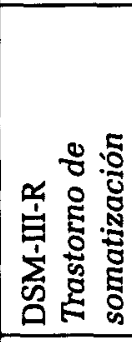 & 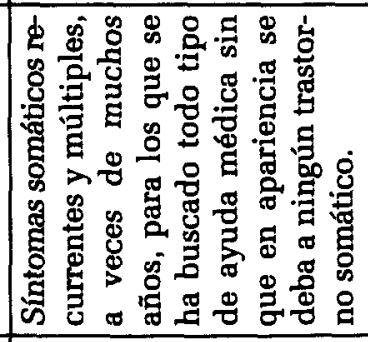 & 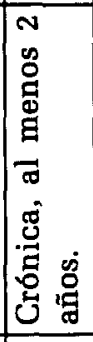 & 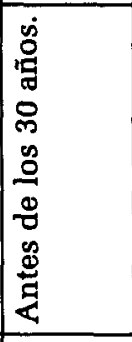 & 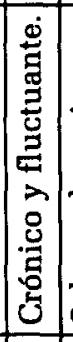 & 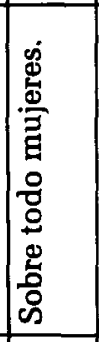 & 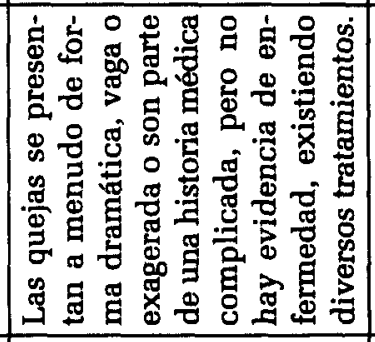 & 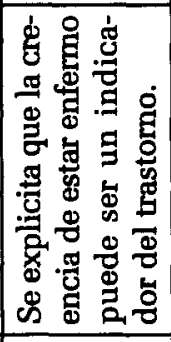 & 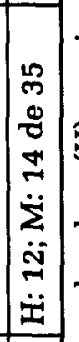 \\
\hline 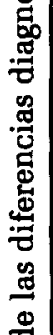 & 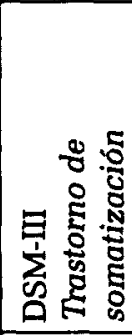 & 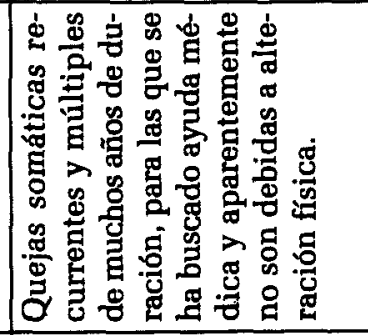 & 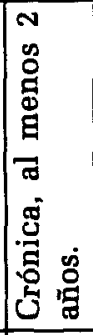 & 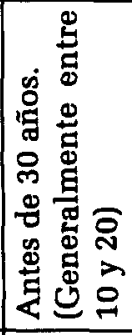 & 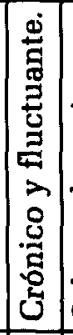 & 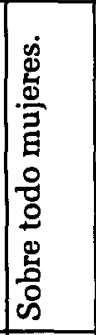 & 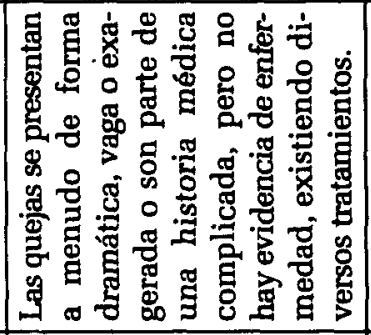 & 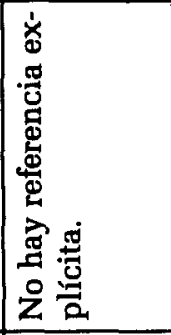 & $\stackrel{m}{\rightarrow}$ \\
\hline & 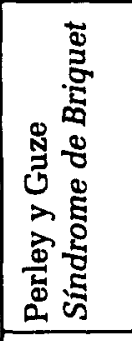 & 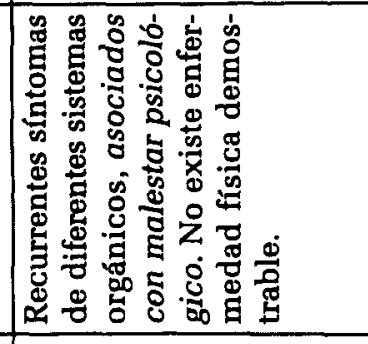 & .8 & 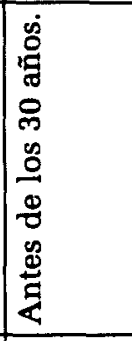 & $\begin{array}{l}0 \\
0 \\
0 \\
0 \\
0 \\
\end{array}$ & 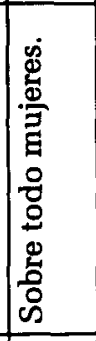 & 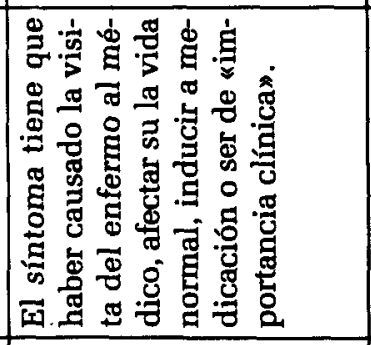 & 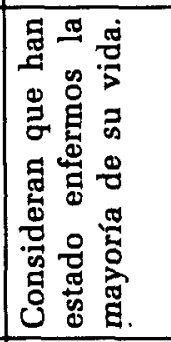 & 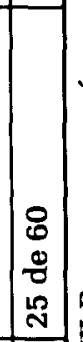 \\
\hline & & 兽 & : & $\mid \stackrel{̊}{\mathscr{U}}$ & जี & 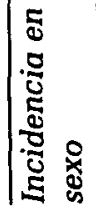 & 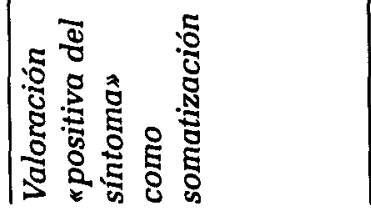 & 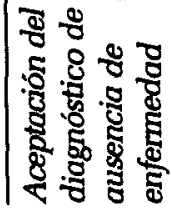 & 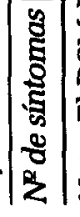 \\
\hline
\end{tabular}


El rechazo a las explicaciones médicas

El rechazo a aceptar las explicaciones médicas que no confirmen la creencia del sujeto de padecer algún tipo de enfermedad, se ha relacionado habitualmente con los distintos trastornos en los que aparecen implicados los sistemas somáticos (somatomorfos), principalmente con Hipocondría, pero como hemos indicado, también con el Trastorno de Somatización, concibiéndolo como "conducta anormal de enfermedad" (Pilowsky, 1978). En la actualidad este tema está sujeto a una gran controversia ya que se pone en cuestión el carácter tranquilizador de las intervenciones médicas y de las explicaciones que se dan en las mísmas (Kellner, 1986; Kirmayer y Taillefer, 1996; Lipowski, 1988; Sharpe, Peveler y Mayou, 1992; Warwick y Salkovskis, 1990).

El problema sobre si los pacientes aceptan o no las explicaciones médicas cuando estas no proporcionan ninguna respuesta que pueda justificar las quejas manifestadas, puede entenderse mejor al tener en cuenta el significado que para muchos pacientes y profesionales de la salud tiene el concepto de «síntoma no explicado médicamente», interpretándose frecuentemente como sinónimo de síntomas imaginarios (Kirmayer y Taillefel, 1996). Ello puede llevar al paciente a realizar denodados esfuerzos para que se admita como «real» algo que, sin duda, personalmente está experimentando como tal, con el consecuente deterioro de la relación médico paciente (Sharpe et al., 1992).

Esta tendencia a interpretar como imaginarios o incluso inventados aquellos síntomas para los que no se encuentra una clara causa orgánica, puede ser explicada como una consecuencia de la tradición epistémica dualista, ya referida anteriormente, que considera a la mente y al cuerpo como entidades claramente sepa- radas (Kirmayer y Taillefer; 1996; Valdés, 1991). A partir de la misma se deduce que un trastorno que no sea somático, taxativamente ha de ser considerado como psíquico, resultando más lícito atribuir a síntomas físicos un origen orgánico (médico), que pensar en posibles causas psicológicas, o en una interacción de ambas. Así, se considera deshonesto e incluso excepcional, el que se expresen en términos somáticos problemas que el profesional sitúa en el ámbito psicológico o social (Kirmayer y Taillefer, 1996).

Esta argumentación puede encontrar su apoyo en los trabajos que han puesto de manifiesto que aquellas intervenciones médicas o psicológicas que se han centrado en tranquilizar al paciente, facilitando atribuciones adecuadas sobre sus síntomas, al mismo tiempo que no han dudado en ningún momento de la veracidad y fenomenología de estos, han resultado de gran utilidad, evitando que factores yatrogénicos acentuaran el problema (Bass y Benjamin, 1993; Creed y Guthrie, 1993; Kellner, 1986; Kroenke y Swindle, 2000; Lidbeck, 1997; MacLeod, Matthew y McClelland 1997; Sharpe, et al., 1992; Smith Rost y Khaner, 1995). Con lo expuesto no se quiere dudar de la existencia de pacientes que insisten en atribuir un claro origen orgánico a sus síntomas, pero sí cuestionar la generalización de ésta idea.

\section{Diagnóstico diferencial}

La inespecificidad de los síntomas que constituyen el trastorno de somatización hacen especialmente relevante un detenido establecimiento del diagnóstico diferencial, en el que además de tener en cuenta otros trastornos psiquiátricos, se ponga un especial énfasis en valorar otras enfermedades médicas caracterizadas por síntomas que podrían solaparse con los que se incluyen en la categoría del tras- 
torno de somatización. El establecimiento de un diagnóstico diferencial cobra especial relevancia cuando entendemos los trastornos psiquiátricos de un modo categorial, estructurados a partir de determinadas nosologías. En este epígrafe no pretendemos ser exhaustivos (para más información véanse APA, 1994 y OMS, 1992), y sólo nos centraremos en los aspectos que resultan más controvertidos, que son los que se refieren al trastorno hipocondríaco.

$\mathrm{El}$ aspecto característico del trastorno hipocondríaco es el miedo, la preocupación o la idea de padecer una enfermedad grave (APA, 1994). El DSM-IV establece que cuando el miedo o preocupación ocurren en el transcurso de un trastorno de somatización no debe hacerse el diagnóstico de hipocondría. En nuestra opinión, este requisito induce a confusión ya que establecer únicamente el diagnóstico de trastorno de somatización cuando se presentan conjuntamente síntomas de somatización e hipocondría, parece presuponer que la presencia de quejas somáticas recurrentes va unido al miedo o preocupación por padecer una enfermedad grave, es decir, que de alguna forma, la somatización implica preocupaciones hipocondríacas. Por otro lado, esto último, no se deduce de los criterios diagnósticos del trastorno de somatización, ya que están configurados con independencia de la presencia o no de convicción de enfermedad o preocupación por ésta. En síntesis, parecería más adecuado establecer ambos diagnósticos cuando se cumplan criterios para el trastorno de somatización conjuntamente con los de hipocondría.

El matiz diferenciador entre hipocondría y trastorno de somatización referido por la CIE-10 resulta especialmente interesante. Por un lado se indica que en el trastorno de somatización se pone un mayor énfasis sobre los síntomas en sí mismos y sus efectos aislados, mientras que en la hipocondría se presta más atención a la enfermedad subyacente. Y por otro, que en la somatización se solicita tratamiento para paliar el malestar, mientras que en la hipocondría las exploraciones médicas tienen como objetivo descartar la presencia de una enfermedad grave. En la CIE-10 no se plantea la posibilidad de que dentro de un trastorno de somatización se den preocupaciones hipocondríacas, pues en este caso se hablaría de trastorno hipocondríaco. Esto queda más claro cuando observamos que la hipocondría se define como «la preocupación persistente por la posibilidad de tener una o más enfermedades somáticas graves progresivas, puesta de manifiesto por la presencia de quejas somáticas persistentes o por preocupaciones persistentes sobre el aspecto físico» (p. 205). Es decir, ante la presencia de quejas somáticas recurrentes, unidas a preocupaciones hipocondríacas, se prioriza el diagnóstico de hipocondría.

\section{Epidemiología}

Tradicionalmente se ha considerado que la presencia de "síntomas funcionales» constituía un porcentaje muy elevado de las quejas manifestadas por los pacientes en las consultas de los médicos de atención primaria, estimándose prevalencias entre un 20 y un $84 \%$ (Kellner, 1985; Smith et al., 1995). En población española Lobo, García Campayo, Campos, Marcos y Pérez-Echeverría (1996) informan que la prevalencia de quejas no relacionadas con ninguna enfermedad conocida era del $25-30 \%$. Por otra parte, se ha indicado que entre un $60-80 \%$ de la población general ha padecido en algún momento de su vida un síntoma de estas características a lo largo de una semana (Kellner, 1985). Más específicamente, dentro del trastorno de somatización, se 
ha concluido generalmente que el riesgo estimado a lo largo de la vida de padecer el trastorno en mujeres se sitúa entre un 0,2 y un $2 \%$ (APA, 1995; Chorot y Martínez, 1995; Kirmayer y Taillefer, 1996; Martin y Yutzy, 1996), hallándose tasas inexistentes o muy bajas en hombres, inferiores al 0,2\% (APA, 1995).

De entre los estudios recientes que han intentado dar cuenta de la actual prevalencia del trastorno, hay dos que son especialmente relevantes. En primer lugar, los resultados del trabajo de Gureje, Simon, Ustum y Goldberg (1997), procedentes de un estudio epidemiológico realizado por la OMS en 14 países, incluyendo una amplia diversidad cultural. La prevalencia del trastorno de somatización en este estudio es de un $2,8 \%$, pero existen puntuaciones extremas en algunas de las muestras: concretamente la prevalencia es de un $17,7 \%$ en Santiago de Chile, frente al 0,1\% de Nagasaki (Japón) y Verona (Italia). Un dato también a destacar es el referido a las diferencias entre sexos, pues aunque la presencia del trastorno es significativamente mayor en mujeres que en hombres (un 1,9\% frente a un $3,3 \%$ ), estas diferencias no son tan grandes como habitualmente se ha supuesto.

El otro trabajo al que nos referíamos previamente (Faravelli et al., 1997) resulta especialmente interesante debido a sus cuidados criterios epidemiológicos y de investigación, en concreto en lo que se refiere a la representatividad de la muestra y la metodología de evaluación de los síntomas. La prevalencia del trastorno de somatización, según este estudio, es del $0,7 \%$ a partir de criterios DSM-III-R, siendo mujeres el $100 \%$ de los diagnósticos positivos. Destaca también que el trastorno somatoforme indiferenciado (TSI) tiene una prevalencia del $13,4 \%(75,2 \%$ de . mujeres). Por otra parte la comorbilidad hallada con otros trastornos era bestante baja. Finalmente, el número de personas que presentan algún tipo de síntoma somático no explicado fue del $33 \%$.

A tenor de la divergencia de resultados parece evidente que, a pesar de los esfuerzos que se han realizado en los últimos años para intentar ofrecer datos fiables de la epidemiología del trastorno, son muchas las lagunas que todavía quedan por resolver. El análisis de estudios epidemiológicos recientes, como los mencionados, pone de manifiesto que muchas de las discrepancias tienen su origen en la utilización de criterios dispares, tanto en lo concerniente a aspectos metodológicos, como en los referidos a la evaluación y el diagnóstico.

\section{Conceptuaciones alternativas del trastorno de somatización}

El trastorno de somatización y el TSI son los dos síndromes propuestos por el DSM-IV a la hora de plantear que la presencia de sintomatología médica no explicada médicamente tienen entidad diagnóstica. Hay varios autores que han cuestionado la representatividad de ambos categorías, considerando que ni el trastorno de somatización, por ser excesivamente excluyente, ni tampoco el TSI, por su inespecificidad - su diagnóstico sólo se da tras la exclusión de otros trastornos somatomorfos- resultan demasiado útiles para dar cuenta de la verdadera incidencia y repercusión de la patología de los pacientes que tradicionalmente han sido denominados como somatizadores (Escobar et al., 1987, 1998; Gureje et al., 1997; Hiller et al., 1997; Katon et al., 1991; Kroenke et al., 1997).

Dentro de la literatura reciente, ha habido diversos intentos de ofrecer una concepción alternativa y más operativa del Trastorno de Somatización. Vamos a referirnos a tres de ellos: la propuesta de una modificación de los criterios de la CIE-10 (Tomasson et al., 1993), la defini- 
ción abreviada del trastorno de somatización (Escobar et al., 1987; 1998) y el trastorno multisomatoforme (Kroenke et al., 1997). Respecto a la primera propuesta, el grupo de Tomasson ha sugerido una versión modificada de los criterios de investigación para el trastorno de somatización de la CIE-10, que consiste fundamentalmente en la eliminación del criterio referido al rechazo de la explicación médica y en hacer hincapié en la evaluación de síntomas. Estos autores encontraron un elevado solapamiento diagnóstico entre estos criterios y las diferentes propuestas del DSM, lo que les indujo a proponer esta conceptuación como "una alternativa razonable para identificar un grupo de pacientes polisintomáticos" (p.314), facilitando un diagnóstico más sencillo (se evalúan 14 síntomas frente a los 34 del DSM-IV).

Escobar y colaboradores $(1987,1989)$ propusieron una definición "abreviada» del trastorno de somatización, también denominada «índice de síntomas somáticos», con el fin de ofrecer una definición más operativa del concepto de somatización y que fuera de utilidad en atención primaria. Para estos autores la noción tradicional en la clínica del paciente «somatizador» no estaba adecuadamente reflejada en las nosologías psiquiátricas al uso, siendo además ampliamente utilizada, tanto en un marco clínico como de investigación, una noción muy inespecífica de somatización. El criterio diagnóstico para estos autores lo constituye la presencia de 4 síntomas somáticos en hombres y 6 en mujeres. Los pacientes que cumplían estos criterios puntuaban alto en número de visitas médicas innecesarias o no programadas, interferencia en el funcionamiento normal, consumo de medicación, junto a otras variables de significación clínica (Escobar et al., 1987). Esta definición «abreviada» parece haber tenido cierta aceptación y repercusión en la literatura (p.ej., Gureje et al., 1997; Hiller et al., 1997; Katon et al., 1991; Kirmayer y Taillefer, 1996; Lobo et al., 1996; Martin y Yutze, 1996; Postergijs et al., 1996).

Según Escobar et al., (1998) las características psicopatológicas y de comorbilidad de este constructo son muy similares a las encontradas con los criterios para la definición "completa" del trastorno, con la importante diferencia de que la prevalencia de la definición «abreviada» en la población general, es aproximadamente 100 veces más frecuente. Se encontró que el $22 \%$ de los sujetos evaluados en atención primaria, cumplían los criterios diagnósticos completos. Además, utilizando el Composite International Diagnostic Interview (CIDI), que evalúa la presencia de hasta un total de 40 síntomas, se constató una diferencia significativa entre sexos, siendo mayor el número de mujeres $(24,9 \%)$ que el de hombres $(18,4 \%)$. Kilesly, Golberg y Simon (1997) proporcionaron resultados similares. Es también relevante el hecho de que el $64 \%$ de estos pacientes han presentado algún trastorno psiquiátrico a lo largo de su vida $(37,5 \%$ si se trata de trastorno depresivo). Además, la comorbilidad de pacientes hipocondríacos con estos criterios de somatización es muy elevada.

Como tercera propuesta encontramos el «trastorno multisomatoforme» (Kroenke et al., 1997), que pretende ofrecer una alternativa operativa y fácil de evaluar del TSI, síndrome que, según estos autores «nunca ha sido validado». Es definido por la presencia de 3 o más síntomas somáticos de un listado de los 15 síntomas más frecuentes en las consultas médicas (los que constituyen un $90 \%$ de las visitas), a partir del análisis de los datos del Primary care evaluation of mental disorders 1000 study (Spitzer, Williams y Kroenke, 1994). La prevalencia de estos criterios es del $8,2 \%$ en aten- 
ción primaria (Kroenke et al., 1997), apareciendo en estos pacientes niveles de deterioro significativos -equiparables a los pacientes con diagnóstico de trastorno de ansiedad o depresión- en relación con la calidad de vida manifestada, el número de días de baja, el número de visitas médicas y el grado de enfermedad percibida por el médico.

Los resultados de esta investigación motivan a sus autores a considerar al trastorno multisomatoforme como una alternativa adecuada al TSI, proponiendo unos criterios diagnósticos específicos que puedan sustituir a los de este trastorno en el DSM-IV. Sin embargo, hay algo que llama la atención: aunque los criterios propuestos resultan razonables y parece útil y necesaria una definición más operativa del TSI, el trastorno multisomatoforme no parece compatible con los diagnósticos del DSM-IV, ya que se basa en un listado de síntomas no equiparable a los utilizados en las evaluaciones de este manual diagnóstico.

Para terminar este punto es importante resaltar que estas nuevas conceptuaciones apoyan la propuesta de Katon et al., (1991) acerca de una descripción dimensional del trastorno de somatización. Según estos autores la somatización puede entenderse como un continuo en el que el aumento de síntomas es un indícador de incremento de malestar, incapacitación y conductas de enfermedad inadecuadas. Otros trabajos, como el de Simon y VonKorff (1991) apoyan esta misma idea.

Finalmente quisiéramos mencionar que la elevada comorbilidad, ya mencionada, entre somatización y otras patologías psiquiátricas, invita a preguntarse detenidamente por la etiología de estos síntomas, por el significado de su coexistencia con otros trastornos psiquiátricos, y por el sentido que, en ocasiones, puede tener resaltar como entidad nosológica la presencia aislada de esos síntomas.

\section{CONCLUSIONES}

Es evidente que no todos los síntomas que han sido considerados como somatizaciones pueden interpretarse de la misma forma. Los diferentes contextos en los que pueden aparecer y el gran número de interpretaciones que sobre diversos síntomas somáticos pueden hacerse cuando éstos no aparecen asociados a una clara patología física, invita a ser cauteloso en las inferencias y significados que les otorguemos. En este sentido nos parece conveniente optar por una conceptuación preferentemente descriptiva, que aunque en ocasiones puede empobrecer nuestro conocimiento de los síntomas, puede evitar que caigamos en el error de generalizar y simplificar la interpretación de los mismos. Además, mantener una definición puramente descriptiva y fenomenológica de un síntoma no quiere decir que cuando exista una explicación plausible en un contexto y dirección determinadas, no podamos admitirla. De este modo, abogar por no generalizar una definición de los síntomas somáticos no explicados médicamente como un lenguaje de expresión de un conflicto psicológico, no quiere decir que no podamos admitir, cuando fuera oportuno, esta interpretación como un heurístico de gran validez.

Desde una perspectiva biopsicosocial (Belloch y Olabarría, 1993) y partiendo de un punto de vista multicausal a la hora de aproximarnos al estudio de la somatización, la propuesta de un reciente trabajo sobre la etiopatogenia de la somatización (Kapfhammer, 2001) puede ser esclarecedora. Dice así: "Para comprender la etiopatogenia de la somatización, resulta obligado recurrir a un modelo multifactorial. Por lo tanto, deben tenerse en cuenta diferentes aspectos de genética, neurología, neurofisiología, psicofisiología, endocrinología, psicología de la personalidad, psicología de la per- 
cepción y cognición, aprendizaje social, conocimiento de la enfermedad, concepción de la enfermedad, eventos vitales, estresores psicosociales crónicos, ausencia de habilidades de afrontamiento, pérdida de apoyo social, el sistema de refuerzo social, comorbilidad de trastornos psiquiátricos, traumas psicológicos y aspectos psicodinámicos» (Kapfhammer, 2001). Sin necesidad de ser tan exhaustivos, esta reflexión puede advertirnos de lo peligroso que pueden resultar muy a menudo las simplificaciones a la hora de interpretar los síntomas definidos como «somatizaciones». En todo caso, el análisis individualizado del papel que pueden estar jugando los síntomas (y la preocupación por los mismos) en aspectos tales como el estado de ánimo del paciente, su red social, o su rendimiento en diversas áreas del funcionamiento cotidiano, nos puede permitir establecer hipótesis ad hoc adecuadas al caso de que se trate y, a partir de ahí, poder diseñar estrategias de intervención adecuadas. Al fin y al cabo, eso y no otra cosa, es lo que para el psicólogo clínico significa "evaluar», tarea mucho más compleja y desde luego, personalizada, que la mera etiquetación diagnóstica.

Respecto al trastorno de somatización como categoría diagnóstica es importante resaltar que, aunque se ha presentado desde hace bastante tiempo como una entidad nosológica bien delimitada y estable (Yutzy et al., 1995), son varias las voces críticas que cuestionan su validez y utilidad clínica (p.ej. Escobar et al., 1987; 1998), e importantes las diferencias que presentan las distintas definiciones del trastorno (DSM y CIE). Todo ello plantea numerosas dudas sobre la claridad conceptual con la que a menudo se presenta el trastorno. Si se pretende que resulte útil como categoría diagnóstica, tendrán que hacerse esfuerzos para clarificar algunas cuestiones que en la actualidad resultan controvertidas. En nuestra opinión, son especialmente importantes las siguientes:

1. Clarificar si puede atribuirse a estos pacientes la persistencia de un rechazo a aceptar un diagnóstico negativo sobre los síntomas. Si la respuesta fuese afirmativa, sería necesario especificar la naturaleza e intensidad de este rechazo, aclarando las similitudes y diferencias con el trastorno hipocondríaco.

2. Llevar a cabo una más clara delimitación de la naturaleza y características de aquellos síntomas que son entendidos como somatizaciones, lo que evitaría en muchas ocasiones la confusión diagnóstica.

3. Profundizar sobre la utilidad y representatividad del trastorno, según viene definido en las actuales clasificaciones nosológicas, planteándose la posibilidad de elaborar definiciones que resulten más operativas y que expliquen mejor el conjunto de pacientes entendidos como somatizadores. En este sentido es útil valorar la idoneidad de propuestas alternativas como las descritas más arriba (Escobar et al., 1987; Kroenke et al., 1997; Tomasson et al., 1993)

El aumento del interés sobre el estudio de los trastornos somatomorfos que parece estar produciéndose en los últimos años, y que se refleja no solo en el aumento de artículos científicos sobre el tema sino sobre todo en la creación de unidades de estudio de los trastornos somatomorfos en el ámbito hospitalario - como la del Hospital Miguel Servet de Zaragoza, dirigida por el Dr. García Campayo-, invita a ser optimistas respecto al avance, en un futuro próximo, de nuestro conocimiento sobre este difícil y controvertido trastorno.

\section{REFERENCIAS BIBLIOGRÁFICAS}


trastornos mentales ( $3^{\mathrm{a}}$ ed.). Barcelona: Masson.

American Psychiatric Association (1988). Manual diagnóstico y estadístico de los trastornos mentales ( $3^{\mathrm{a}}$ ed. revisada). Barcelona: Masson.

American Psychiatric Association (1995). Manual diagnóstico y estadístico de los trastornos mentales ( $4^{\mathrm{a}}$ ed.). Barcelona: Masson.

Bass, C. y Benjamin, M. (1993). The managament of chronic somatization. British Journal of Medical Pshychology, 162, 472-480.

Belloch, A. y Olabarría, B. (1993). El modelo bio-psico-social: Un marco de referencia necesario para el psicólogo clínico. Clinica y Salud, 4, 181-190.

Bridge, R.N. y Golberg,D.P. (1985). Somatic presentation of DSM-III psychiatric disorders in primary care. Journal of Psychosomatic Research, 29, 563-569.

Bridge R. N., Golberg, D.P., Evans, B. y Sharpe, T. (1991). Determinants of somatization in primary care. Psychological Medicine, 21, 473-483.

Brown, F.W. y Smith, O.R. Jr. (1991). Diagnostic concordance in primary care somatization disorder. Psychosomatics, 32,191195.

Chorot, P., Martínez, P. (1995). Trastornos somatoformes. En: A. Belloch, B. Sandín y F. Ramos (Eds.), Manual de psicopatología, Vol. 2 (pp. 225-266). Madrid: McGraw Hill.

Cloninger C.R., Martin, R.L., Guze, S.B. y Clayton, P.J. (1986). A prospective followup and family study of somatization in men and woman. American Journal of Psychiatry, 143, 873-878.

Creed, F. y Guthrie, E. (1993). Techniques for interviewing the somatising patients. British Journal of Psychiatry, 162, 467-471

Deary, I.J., Scott, S. y Wilson, J.A. (1997). Neuroticism, alexithymia and medically unexplained symtoms. Personality and Individual Differences, 22, 551-564.

Escobar, J.I., Burnam, M.A., Karno, M., Forsythe, A. y Golding, J.M. (1987). Somatization in the comunity. Archives of General Psychiatry, 44, 713-718.

Escobar, J.I., Rubio-Stipec, M. y Canino, G. (1989). Somatic Symtoms Index (SSI): A new and abridged somatization construct:
Prevalence and epidemiological correlates in two larges community samples. Journal of Nervous and Mental Diseases, 177, 140146.

Escobar, J.I., Waitzkin, H., Silver, R.C., Gara, M. y Holman, A. (1998). Abridged somatization: a study in primary care Psychosomatic Medicine, 60, 466-472

Faravelli, C., Salvatori, F., Galassi, L., Aiazzi, L., Drei, C. y Cabras, P. (1997). Epidemiology of somatoform disorders: a community survey in Florence. Social Psychiatry and Psychiatric Epidemiology, 32, 24-29.

Feighner, J. P., Robins. E., Guze, S. B., Woodruff, E., Winokun, M. y Muñoz, M. (1972). Diagnostic criteria for use in psychiatry research. Archives of General Psychiatry, 26, 57-63.

Ford, C. V. (1983). The somatizing disorders: Illness as a way of life. Nueva York: Elsevier.

Freud, S. (1993) La formación de síntomas. En S. Freud (Ed.), Compendio de psicoanálisis. Barcelona: Ediciones Altaya. (Edición original 1916).

García Campayo, J. (1999) Usted no tiene nada. La somatización. Barcelona: Océano.

García-Campayo, J., Campos, R., Marcos, G., Pérez-Echeverría, M., y Lobo, A. (1996a) Somatisation in primary care in Spain. II. Differences between Somatisers and Psychologisers. British Journal of Psychiatry, 168, 344-353.

García-Campayo, J., Sanz, C., Pérez-Echeverría, M., Campos, R. y Lobo, A. (1996b). Screening of somatization disorder: validation of the Spanish version of the Othmer and DeSouza test. Acta Psychiatrica Scandinava, 94, 411-415.

García-Campayo, J., Saez Carrillo, C., Campos, R., Marcos, G., Pérez-Echeverría, M., y Lobo, A. (1995). Trastomo por somatizacíón en atención primaria: aspectos cínicos diferenciales. Medicina Clinica, 105, 728-733.

Gureje, O., Simon, G., Ustum, T.V. y Golberg, D.P. (1997). Somatization in cross-cultural Pepective: A WHO Study in primary care. American Journal of Psychiatry, 154, 989995.

Guze, S.B. y Perley M.J (1963). Obsevation on the natural history of hysteria. American Journal of Psychiatry, 119, 960-965. 
Hällström, P.M. (1998). Depressive disorders among somatizing patients in primary health care. Acta Psychiatrica Scandinavica, 98, 187-192.

Hiller, W., Rief, W. y Fichter, M.M. (1997). How disabled are patients with somatofom disorders?. General HospitaI Psychiatry, 19, 432-438.

Kapfhammer, H.P.(2001). Somatization and somatoform disorders: etiopathological models. Fortschritte der Neurologe-Psychiatrie, 69, 58-77.

Katon, W. Kleinman, A. y Rosen, G. (1982). Depresion and somatization: A review. Part I. American Journal of Medicine, 72, 127135.

Katon, W., Lin, E., Von Korff, M., Russo, J., Lipscomb, P. y Bush, T. (1991). Somatization: A Spectrum of Severity. American Journal of Psychiatry, 148, 34- 40.

Kellner, R (1994). Psychosomatic syndromes, somatic somatization and somatoform disorders. Psychotherapy and Psychosomatics, 61, 4-24.

Kellner, R. (1985). Funcional somatic symptoms and hypochondriasís. Archives of General Psychiatry, 42, 821-833.

Kellner, R. (1986) Somatization and hypochondriasis. New York, Praeger Special Studies.

Kellner, R. (1991). Psychosomatic Syndromes and Somatic Symptoms. Washington DC. American Psychiatric Press.

Kirmayer, L. J. y Robbins, J. M. (1996). Patients who somatize in primary care: A longitudinal study of cognitive and social characteristics. Psychological Medicine, 26, 937-51

Kirmayer, L. J. y Taillefer, S. (1996) Somatoform disorders. En S.M. Turner y M. Hersen (Eds.), Adult Psychopatology and Diagnosis (pp.304-328). Nueva York: Wiley.

Kisely, S., Golberg, D. y Simon G. (1997). A compararison between somatic symtoms with and without clear organic cause: results of an intenational study. Psychological Medicine, 27, 1011-1019.

Kleinman, A. y Kleinman, J. (1986). Somatization: the interconnections among culture, depressive experiences, and the meaning of pain. En A. Kleinman, B. Good y
C.A. Berkeley (Eds.), Culture and Depresion (pp. 429-490). CA: Univerity of California Press.

Kroenke, K. y Swindle, R. (2000). Cognitivebehavioral therapy for somatization and symptom syndromes: a critical review of controlled clinical trials. Psychotheraphy and Psychosomatics, 69, 205-215.

Kroenke, K., Spitzer, R. L., deGruy III, F. V., Hahn, S. R., Linzer, M., Williams, J. B., Brody, D. y Davies, M. (1997). Multisomatoform disorder. Archives of General Psychiatry, 54, 352-358.

Laplanche, J y Pontalis, J.B (1994). Diccionario de Psicoanálisis. Colombia: Labor.

Lidbeck, J. (1997). Group therapy for somatization disorders in general practice: effectiveness of a short cognitive-behavioural treatment model. Acta Psychiatrica Scandinava, 96,14-24.

Lipowski, Z. J. (1988). Somatization: The concept and its clinical application. American Journal of Psychiatry, 145, 1358-1368.

Liu, 0., Clark, M.R. y Eaton, W.W. (1997). Structural factor analyses for medically unexplained somatic symptoms of somatization disorder in the Epidemiologic Catchment Area study. Psychological Medicine, 27, 617-26.

Lobo, A., García-Campayo, J., Campos, R., Marcos, G., y Pérez-Echeverría, M. (1996). Somatisation in Primary Care in Spain. I. Estimates of prevalence and clinical characteristics. British Journal of Psychiatry, 168, 344- 353.

Martin, R. L. y Yutzy, S. H. (1996) Trastornos de somatización. En R.E. Hales, S.C. Yudofsky, y J.P.Talbott (Eds.), Tratado de psiquiatría (2a Edición; pp. 350-396). Barcelona: Áncora.

Mayou, R. (1976). The nature of bodily symtoms. British Journal of Psychiatry, 129, 5560.

McLeod, C.C., Matthew, A.B. y McClelland, D.C. (1997). Treatment of somatization in primary care. General Hospital of Psychiatry, 19, 251-258.

Mechanic, D. (1962). The concept of illness behavior. Journal of Chronic Diseases, 15, 189-194.

Moliner, M. (1998). Dicionario de uso del español (2 ${ }^{a}$ Edición). Madrid: Gredos. 
Ohmer, E. y DeSouza, C. (1985). A screnning test for somatization disorder. American Journal of Psychiatry, 142, 1146-1149.

Organización Mundial de la Salud (OMS) (1992). Trastornos mentales y del comportamiento, $10^{g}$ edición. (CIE-10). Madrid: Meditor.

Organización Mundial de la Salud (OMS) (1993). Criterios diagnóstico y de investigación de los trastornos mentales y del comportamiento (CIE-10). Madrid: Meditor.

Perley, M.J. y Guze, S. B. (1962). Hysteria, the stability and usefulness of clinical criteria. New England Journal of Medicine, 266, 421-426.

Pilowsky, L. (1969). Abnormal illness behavior. British Journal of Psychology, 42, 347351.

Pinillos, J. L. (1975). Principios de psicologia. Madrid: Alianza Editorial.

Portegijs, P.J., Van der Horst, F.G., Proot, M.I., Kraan, B.F., Gunther, N.C. y Knottnerus, J.A. (1996). Somatization in frequent attenders of general practice. Social Psychiatry and Psychiatric Epidemiology, 31, 29-37.

Robbins, J.M. y Kirmayer, L.J. (1991). Atributions of common somatic symptoms. Psychological Medicine, 21,1029-1045.

Rodriguez Marín, J. (1995). Psicología social de la salud. Madrid: Sintesis.

Seco, M., Andrés, O., y Ramos, G. (1999). Diccionario del español Actual. Madrid: Aguilar.

Sharpe, M., Peveler, R. y Mayou. R. (1992). The psychological treatment of patients with functional somatic symptoms: A practical guide. Journal of Psychosomatic Research, 36, 515-529.

Simon, G. y Gureje, O.(1999).Stability of somatization disorder and somatization symptoms among primary care patients. Archives of General Psychiatry, 56,90-95.

Simon, O. y Von Korff, M. (1991). Somatization and psychiatric disorder in the NIMH Epidemiological Catchment Area study. American Journal of Psychiatry, 148, 14941500.

Smith, G., Rost, K. y Kashner, M. (1995). A trial of effect of a standardized psychiatric consultation on health outcomes and costs in somatízing patients. Archives of General Psychiatry, 52, 238-243.

Spitzer, R., Williams, J., y Kroenke, K. (1994). Utility of a new procedure for diagnosing mental disorders in primary care: The PRIME-MD 1000 Study. JAMA, 272,17491756.

Tomasson, K., Kent, D. y Coryell, W. (1993). Comparision of four diagnostic systems for somatization disorder. Acta Psychiatrica Scandinavica, 88, 311-315

Valdés, M. Trastornos psicosomáticos. (1991). En Vallejo, J. (Ed.) Introducción a la psicopatología y la psiquiatria (pp. 520-529). Barcelona: Masson.

Vallejo, J. (Ed.) (1991). Introducción a la psicopatología y la psiquiatría. Barcelona: Masson.

Vieta, E., Gasol, M., Blanch, J. y Cirera, E (1996). Somatización en atención primaria. Medicina Clinica, 107,116-117.

Warwick, H. y Salkovskis, P. (1990) Hypochondriasis. Behaviour Research and Therapy, 28, 105-117.

Yutzy, S. H., Cloninger C. R., Guze, S. B., Martin, R. L., Pribor, E., Kathol, R., Smith, R., y Strain, J. (1995). DSM-IV Field trial: testing a new proposal for somatization disorder. American Journal of Psychiatry, 152, 97-101. 